\title{
Coherence of Nitrogen-Vacancy Electronic Spin Ensembles in Diamond
}

\section{Citation}

Stanwix, Paul L., Linh M. Pham, Jeronimo R. Maze, David Le Sage, Tsun K. Yeung, Paola Cappellaro, Philip R. Hemmer, Amir Yacoby, Mikhail D. Lukin and Ronald L. Walsworth. 2010. Coherence of nitrogen-vacancy electronic spin ensembles in diamond. Physical Review B 82(20): 201201.

\section{Published Version}

doi:10.1103/PhysRevB.82.201201

\section{Permanent link}

http://nrs.harvard.edu/urn-3:HUL.InstRepos:8951177

\section{Terms of Use}

This article was downloaded from Harvard University's DASH repository, and is made available under the terms and conditions applicable to Other Posted Material, as set forth at http:// nrs.harvard.edu/urn-3:HUL.InstRepos:dash.current.terms-of-use\#LAA

\section{Share Your Story}

The Harvard community has made this article openly available.

Please share how this access benefits you. Submit a story.

\section{Accessibility}




\title{
Coherence of nitrogen-vacancy electronic spin ensembles in diamond
}

\author{
P. L. Stanwix, ${ }^{1,2}$ L. M. Pham, ${ }^{3}$ J. R. Maze, ${ }^{4,5}$ D. Le Sage, ${ }^{1}$ T. K. Yeung, ${ }^{3}$ P. Cappellaro, ${ }^{6}$ P. R. Hemmer, ${ }^{7}$ A. Yacoby, ${ }^{4}$ \\ M. D. Lukin, ${ }^{4}$ and R. L. Walsworth ${ }^{1,4, *}$ \\ ${ }^{1}$ Harvard-Smithsonian Center for Astrophysics, Cambridge, Massachusetts 02138, USA \\ ${ }^{2}$ School of Physics, University of Western Australia, Crawley, Western Australia 6009, Australia \\ ${ }^{3}$ School of Engineering and Applied Sciences, Harvard University, Cambridge, Massachusetts 02138, USA \\ ${ }^{4}$ Physics Department, Harvard University, Cambridge, Massachusetts 02138, USA \\ ${ }^{5}$ Facultad de Física, Pontificia Universidad Católica de Chile, Casilla 306, Santiago, Chile \\ ${ }^{6}$ Department of Nuclear Science and Engineering, Massachusetts Institute of Technology, Cambridge, Massachusetts 02139, USA \\ ${ }^{7}$ Department of Electrical and Computer Engineering, Texas A\&M University, College Station, Texas 77843, USA
}

(Received 13 October 2010; published 17 November 2010)

\begin{abstract}
We present an experimental and theoretical study of electronic spin decoherence in ensembles of nitrogenvacancy $(\mathrm{NV})$ color centers in bulk high-purity diamond at room temperature. Under appropriate conditions, we find ensemble NV spin coherence times $\left(T_{2}\right)$ comparable to that of single NV with $T_{2}>600 \mu \mathrm{s}$ for a sample with natural abundance of ${ }^{13} \mathrm{C}$ and paramagnetic impurity density $\sim 10^{15} \mathrm{~cm}^{-3}$. We also observe a sharp decrease in the coherence time with misalignment of the static magnetic field relative to the $\mathrm{NV}$ electronic spin axis, consistent with theoretical modeling of $\mathrm{NV}$ coupling to a ${ }^{13} \mathrm{C}$ nuclear-spin bath. The long coherence times and increased signal-to-noise provided by room-temperature NV ensembles will aid many applications of NV centers in precision magnetometry and quantum information.
\end{abstract}

DOI: 10.1103/PhysRevB.82.201201

PACS number(s): 76.30.Mi, 03.65.Yz, 78.55.-m

The nitrogen-vacancy (NV) color center in diamond is a promising solid-state platform for studying the quantum dynamics of spin systems. Experiments demonstrating spinstate control and manipulation using single NV centers and their nuclear environment ${ }^{1-5}$ have inspired recent work toward quantum information applications of NV diamond, as well as applications to precision magnetic field sensing, ${ }^{6-8}$ including the life sciences. ${ }^{9,10}$ Common to all proposals is a desire for long NV electronic spin coherence times, ideally at room temperature, and high signal-to-noise of the NV spinstate-dependent optical fluorescence.

Electronic spin decoherence of the NV center $(S=1)$ is governed by interactions with the surrounding bath of nuclear and paramagnetic spins [Fig. 1(a)]. In high-purity diamond, decoherence is dominated by ${ }^{13} \mathrm{C}$ nuclear spins $(I=1 / 2),{ }^{11}$ which are dispersed through the crystal with a natural abundance of $1.1 \%$. Electronic spin coherence times $\left(T_{2}\right)$ longer than $600 \mu \mathrm{s}$ at room temperature have been observed for individual $\mathrm{NV}$ centers in this type of sample. ${ }^{7,12}$ $T_{2}$ can be increased by isotopically enriching the diamond; ${ }^{12}$ for example, $T_{2}>1.8 \mathrm{~ms}$ at room temperature has been observed in isotopically enriched ultrapure diamond with $0.3 \%{ }^{13} \mathrm{C} .{ }^{13}$

In addition to long electronic spin $T_{2}$, enhancing the signal-to-noise of NV spin-state-dependent fluorescence is of significant benefit to precision magnetometry. To that end, ensembles of NV centers have been proposed, ${ }^{6,14}$ for which signal-to-noise ideally increases as the square root of the number of NVs in the ensemble. The use of large spin ensembles with good coherence times is also important for collective quantum memories. ${ }^{15-17}$ Increasing the density of NV centers, however, is accompanied by an increased density of residual nitrogen paramagnetic impurities, which can become the dominant source of NV spin decoherence, reducing $T_{2}$ below the limit set by ${ }^{13} \mathrm{C}$ nuclear spins alone. ${ }^{18-20}$
Furthermore, the large variation in coherence times for individual NV electronic spins, due to the random distribution of ${ }^{13} \mathrm{C}$ near each NV center, can also affect ensemble coherence. Therefore, a detailed understanding of decoherence mechanisms affecting NV centers-both single NV and ensembles-accompanied by the development of control techniques to mitigate decoherence is of great importance for

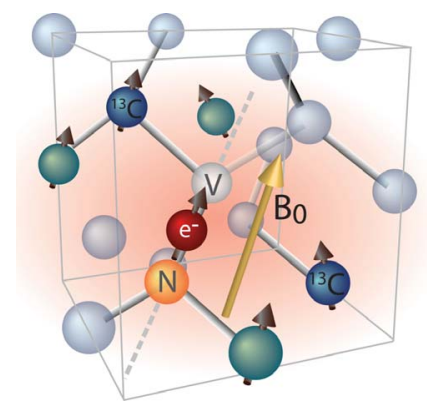

(a)

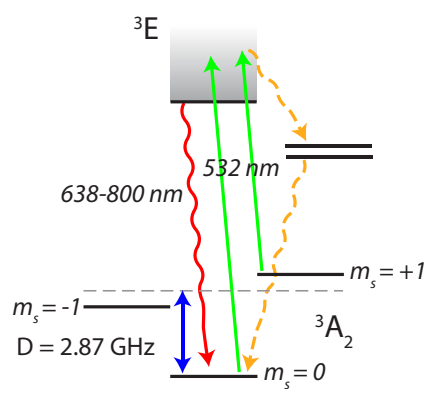

(b)

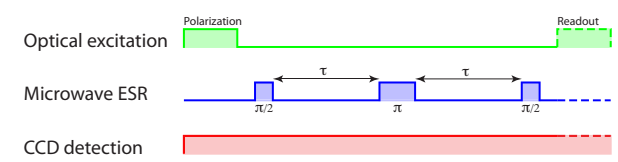

(c)

FIG. 1. (Color online) (a) NV electronic spin axis is defined by nitrogen and vacancy sites, in one of four crystallographic directions. NV orientation subsets in an ensemble can be spectrally selected by applying a static magnetic field, $B_{0}$. Also shown are ${ }^{13} \mathrm{C}$ nuclear spins and other paramagnetic impurities such as substitutional nitrogen. (b) NV center electronic energy-level structure. Spin polarization and readout is performed by optical excitation and fluorescence detection. Ground-state spin manipulation is achieved by resonant microwave excitation. The ground-state triplet has a zero magnetic field splitting $D \simeq 2.87 \mathrm{GHz}$. (c) Hahn-echo experimental sequence used in present measurements. 
both precision measurement and quantum information applications.

Only limited studies of electronic spin decoherence in ensembles of NV centers have been previously reported. At room temperature, ensemble $T_{2}$ times of $58 \mu$ s were measured in chemical-vapor deposition (CVD) diamond; ${ }^{21}$ whereas at low temperature $(<2 \mathrm{~K})$ and high magnetic field ( $>8 \mathrm{~T}$ ), NV ensemble $T_{2}$ of $250 \mu$ s was measured in hightemperature high-pressure type- $1 \mathrm{~b}$ diamond, decreasing to $T_{2}<10 \mu$ s for temperatures $>20 \mathrm{~K}^{22}$

In the present Rapid communication, we report an experimental and theoretical study of the coherence properties of $\mathrm{NV}$ electronic spin ensembles in room-temperature diamond samples of different paramagnetic nitrogen (and consequently NV) concentrations. For a lower nitrogen density sample $\left(\approx 10^{15} \mathrm{~cm}^{-3}\right)$ with natural abundance of ${ }^{13} \mathrm{C}$, we find NV ensemble $T_{2}$ in excess of $600 \mu \mathrm{s}$, comparable to the best results for single $\mathrm{NV}$ center measurements in natural isotopic abundance diamond and an order of magnitude greater than previous room-temperature ensemble measurements. For a higher nitrogen density sample $(\approx 5$ $\times 10^{15} \mathrm{~cm}^{-3}$ ) with natural abundance of ${ }^{13} \mathrm{C}$, we find an $\mathrm{NV}$ ensemble $T_{2} \approx 300 \mu \mathrm{s}$. Furthermore, for both samples we find a sharp decrease in the NV ensemble $T_{2}$ with misalignment of the static magnetic field relative to the NV electronic spin axis being studied, which is consistent with our theoretical modeling of an ensemble of NV electronic spins interacting with a ${ }^{13} \mathrm{C}$ spin bath. ${ }^{11}$

For high-purity diamond, decoherence of single NV electronic spins is dominated by hyperfine interactions with nearby ${ }^{13} \mathrm{C}$ nuclear spins. The contact term of this interaction decreases exponentially with separation; after a few lattice sites it is not larger than a few megahertz. ${ }^{23}$ Meanwhile, the dipolar part of this interaction decreases as $r^{-3}$ and is responsible for the collapses and revivals observed in Hahn-echo measurements of single NV centers. ${ }^{1}$ When the externally applied static magnetic field is aligned with the NV axis, the dipolar field contributions of all ${ }^{13} \mathrm{C}$ nuclei cancel after each $2 \pi$ Larmor precession period of the ${ }^{13} \mathrm{C}$ nuclear spins, as can be measured by the Hahn-echo sequence. ${ }^{1-3}$ Over longer time scales $\left(>600 \mu\right.$ s for natural abundance $\left.{ }^{13} \mathrm{C}\right)$, weak dipole-dipole interactions between ${ }^{13} \mathrm{C}$ nuclei and between ${ }^{13} \mathrm{C}$ and paramagnetic impurities induce fluctuations (flipflops) in the $\mathrm{NV}-{ }^{13} \mathrm{C}$ hyperfine interaction, leading to $\mathrm{NV}$ spin decoherence. The predicted form of this decoherence for the Hahn-echo signal of an individual NV is $\exp \left[-\beta \tau^{n}\right]$, where $n$ is between three and four and the constant $\beta$ depends on details of the relative location of nearby ${ }^{13} \mathrm{C}$ nuclei. ${ }^{11}$ However, when the magnetic field makes a small angle $\theta$ with the NV axis, the Larmor precession frequency $\Omega_{i}$ of individual ${ }^{13} \mathrm{C}$ nuclei is modified by the hyperfine interaction with the NV center to become dependent on $\theta$ and the relative $\mathrm{NV}_{-}{ }^{13} \mathrm{C}$ position $\boldsymbol{r}_{i}: \Omega_{i}=\Omega_{0}+\delta \Omega\left(\theta, \boldsymbol{r}_{i}\right)$. As a consequence, ${ }^{13} \mathrm{C}$ nuclei do not all precess with the same frequency and thus their modulations of the NV spin coherence do not rephase at the same time in the Hahn-echo sequence, inducing NV spin decoherence of the form $\exp \left[-\alpha(\theta) b \tau^{2}\right] .{ }^{11}$ Here $\alpha(\theta)$ describes the misalignment angle dependence of the imperfect Hahn-echo rephasing and $b$ is proportional to the square of the hyperfine interaction between the NV elec- tronic spin and the nearest ${ }^{13} \mathrm{C}$ nuclear spin. For small $\theta$, $\alpha(\theta) \simeq \theta^{2} .24$

Note that the physics of this angle-dependent NV spin decoherence is fundamentally different from previously observed effects for donor-electron spins in semiconductors such as Si:P. ${ }^{25,26}$ For Si:P and similar $S=1 / 2$ systems, the electronic spin-quantization axis points along the external magnetic field; whereas for NV centers, the anisotropy of the electronic distribution causes spin quantization along the NV axis for small magnetic fields $(<500 \mathrm{G})$. In Si:P and similar systems, electron-spin decoherence is dominated by fluctuations of a dipolar-coupled nuclear-spin bath, and is maximized when the magnetic field is aligned along the [111] axis, due to the enhanced flip-flop rate of the nuclear spins arising from the angular dependence of their dipolar coupling. As noted above, NV spin decoherence is minimized when the magnetic field is aligned with the NV axis.

In diamond samples where the concentration of NV centers is low enough that they do not interact significantly with each other and with nitrogen paramagnetic impurities, the ensemble Hahn-echo signal can be considered as the average of many independent signals from individual NV centers. In this case, the ensemble Hahn-echo-signal envelope, $E(\tau)$, can be significantly different from measurements of single NV centers $^{27,28}$ because an ensemble contains a broad distribution of spin coherence lifetimes due to variations in the location of ${ }^{13} \mathrm{C}$ nuclei proximal to individual $\mathrm{NVs}$ in the ensemble

$$
E(\tau)=\int d b \exp \left[-\alpha(\theta) b \tau^{2}\right] f(b) .
$$

Here, $f(b)$ is the probability distribution for the magnitude of the NV-proximal ${ }^{13} \mathrm{C}$ hyperfine-interaction squared in an ensemble. Depending on details of this distribution, the ensemble Hahn-echo-signal envelope can have either a single exponential or Gaussian decay at large times $\tau^{27,28}$

In our experiments we used a custom-built, wide-field-ofview fluorescence microscope to measure the coherence properties of large ensembles of NV centers. The electronic spin state of negatively charged NV centers can be polarized, manipulated and readout using optical and microwave excitation [see Fig. 1(b)]. Spin-state-dependent radiativerelaxation enables NV polarization and readout: NVs initially in $m_{s}=0$ optically cycle between the ground and an electronically excited "bright" state while NVs initially in $\left|m_{s}\right|=1$ have a significant branching to a long-lived metastable "dark" state that nonradiatively relaxes to $m_{s}=0$. Optical excitation is provided by a switched $532 \mathrm{~nm}$ laser source, focused onto the sample with a 20x objective. Fluorescence emanating from the sample is collected back through the same objective, then filtered and imaged onto a charge coupled device (CCD) array. The use of a CCD array allows the sample response to be spatially resolved with resolution $<1 \mu \mathrm{m}$ and field-of-view $>100 \mu \mathrm{m}$. The ensemble of NVs is coherently manipulated on a ground-state electronic spin transition with a resonant microwave magnetic field generated from a loop antenna placed close to the diamond sample, which produces a homogenous microwave $B_{1}$ field over the region of interest. The degeneracy of the 


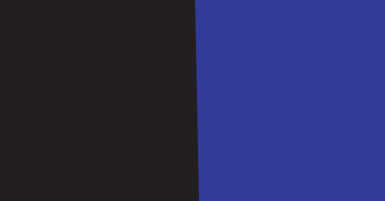

$m_{s}=0$ to $m_{s}= \pm 1$ ground-state spin transitions uniform static $B_{0}$ magnetic field, applied using magnet trimmed by a three-axis set of Helmho cise alignment of $B_{0}$ allows a subset of NVs in corresponding to one of four crystallographic o be spectrally distinguished by their spin transit entation $\theta$ and magnitude $B_{0}$ of the applied $n$ relative to the axis of one class of NV centers from the Zeeman shifts of the NV ground-st spin resonances (ESR) together with the me echo revival frequency, $B_{0}=\omega_{L} / \gamma^{13} C^{29}$

We employed two CVD diamond samples in ments of NV ensembles. Each sample consi diamond substrate $(3 \times 3 \times 0.2 \mathrm{~mm})$ with $<1$ ent nitrogen impurity and $<10^{12} \mathrm{~cm}^{-3} \mathrm{NV} d$ was overgrown with a high $\mathrm{NV}$ content layer The density of NV centers in the thin layer regi $\mathrm{A}$ and $\mathrm{B}$ is approximately $3 \times 10^{13} \mathrm{~cm}^{-3}$ and 1 respectively, as determined by fluorescence normalized to single $\mathrm{NV}$ measurements $\mathrm{m}$ sample using a confocal microscope optimized surements, similar to that used in Ref. 7. T nitrogen impurities in the samples is approxima larger, as determined by the CVD growth proce echo pulse sequence [illustrated in Fig. 1(c)] measure the electronic spin coherence time o semble in each of the two samples. NV cent prepared in the $m_{s}=0$ ground state by applyi laser excitation pulse. The Hahn-echo sequen $-\tau-\pi / 2$ ) was then repeated for a range of free periods $\tau$ (the final $\pi / 2$ pulse in this sequen coherent superposition of $\mathrm{NV}$ spin states into 


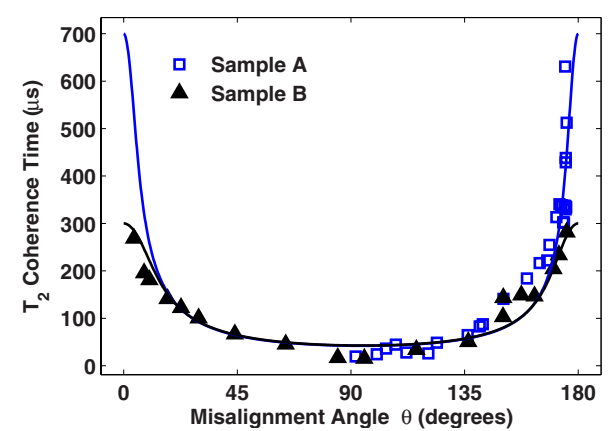

(a)

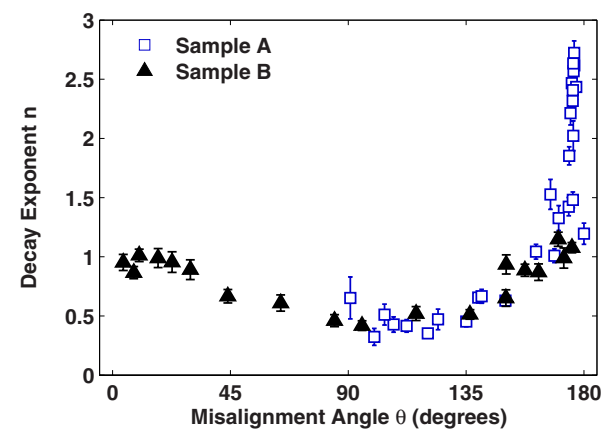

(b)

FIG. 3. (Color online) Summary of Hahn-echo measurements as a function of misalignment angle $\theta$ between the static magnetic field, $B_{0}$, and the NV electronic axis: (a) coherence lifetime $T_{2}$ and (b) decay exponent $n$, determined from fits of the signal envelope to $\exp \left[-\left(\tau / T_{2}\right)^{n}\right]$. Sample A (lower NV and nitrogen concentration) was measured for $\theta$ ranging from $90^{\circ}$ to $180^{\circ}$; sample B (higher NV and nitrogen concentration) was measured for $\theta$ from $0^{\circ}$ to $180^{\circ}$. Solid curve in (a) is the prediction of the NV ensemble decoherence model, as described in the main text.

squared, $f(b)$, was determined by calculating the effect of ${ }^{13} \mathrm{C}$ nuclei placed randomly in the diamond lattice at natural isotopic abundance. For the dominant $\mathrm{NV}^{13} \mathrm{C}$ dipole-dipole interaction, we find that this distribution scales as $f(b)$ $\sim b^{-3 / 2}$ for ${ }^{13} \mathrm{C}$ nuclei within about $10 \mathrm{~nm}$ of a NV center. Thus, $f(b)$ more closely resembles a Lorentzian than a Gaussian distribution, resulting in Hahn-echo-signal envelopes that decay more like a single exponential than a Gaussian at large times (see Fig. 2). Note that for sample B, the experimentally determined decay exponent is $n \simeq 1$ even for $\theta \simeq 0$ [Fig. 3(b)], which could result from interactions between NV spins and the higher density of paramagnetic impurities (nitrogen and NVs) in this sample.

In summary, we demonstrated experimentally that large ensembles of NV centers in high-purity diamond with natural abundance of ${ }^{13} \mathrm{C}$ can have electronic spin coherence lifetimes at room temperature that are comparable to the best measured for single NV centers $\left(T_{2}>600 \mu \mathrm{s}\right)$. We also found a sharp decrease in NV $T_{2}$ as the applied magnetic field is misaligned from the $\mathrm{NV}$ axis, consistent with the predictions of our model for an ensemble of NV electronic spins $(S=1)$ coupled via position-dependent hyperfine interactions to a ${ }^{13} \mathrm{C}$ nuclear-spin bath, leading to imperfect ${ }^{13} \mathrm{C}$ spin-echo revivals and hence, NV decoherence. Our results demonstrate the potential of NV ensembles for applications in precision magnetometry in both the physical and life sciences, combining long electronic spin coherence times at room temperature with the enhanced signal-to-noise ratio provided by many $\mathrm{NVs}$ in the detection volume. ${ }^{6}$ In addition, the demonstrated techniques could be used to increase the coherence time of solid-state quantum memories, such as a $\mathrm{NV}$ electronic spin ensemble coupled to a superconducting resonator ${ }^{15,16}$ or flux qubit. ${ }^{17}$

This work was supported by NIST, NSF, and DARPA. We gratefully acknowledge the provision of diamond samples by Apollo Diamond and technical discussions with Patrick Doering and David Glenn.

\footnotetext{
*rwalsworth@cfa.harvard.edu

${ }^{1}$ L. Childress et al., Science 314, 281 (2006).

${ }^{2}$ T. Gaebel et al., Nat. Phys. 2, 408 (2006).

${ }^{3}$ M. V. G. Dutt et al., Science 316, 1312 (2007).

${ }^{4}$ P. Neumann et al., Science 320, 1326 (2008).

${ }^{5}$ L. Jiang et al., Science 326, 267 (2009).

${ }^{6}$ J. Taylor et al., Nat. Phys. 4, 810 (2008).

${ }^{7}$ J. Maze et al., Nature (London) 455, 644 (2008).

${ }^{8}$ G. Balasubramanian et al., Nature (London) 455, 648 (2008).

${ }^{9}$ C. L. Degen, Appl. Phys. Lett. 92, 243111 (2008).

${ }^{10}$ L. T. Hall et al., Phys. Rev. Lett. 103, 220802 (2009).

${ }^{11}$ J. R. Maze et al., Phys. Rev. B 78, 094303 (2008).

${ }^{12}$ N. Mizuochi et al., Phys. Rev. B 80, 041201 (2009).

${ }^{13}$ G. Balasubramanian et al., Nature Mater. 8, 383 (2009).

${ }^{14}$ V. M. Acosta et al., Phys. Rev. B 80, 115202 (2009).

${ }^{15}$ A. Imamoğlu, Phys. Rev. Lett. 102, 083602 (2009).

${ }^{16}$ Y. Kubo et al., Phys. Rev. Lett. 105, 140502 (2010).

${ }^{17}$ D. Marcos et al., arXiv:1001.4048, Phys. Rev. Lett. (to be published).

${ }^{18}$ E. Van Oort et al., Chem. Phys. 143, 131 (1990).

${ }^{19}$ E. van Oort et al., Chem. Phys. 152, 365 (1991).

${ }^{20} \mathrm{R}$. Hanson et al., Science 320, 352 (2008).
}

${ }^{21}$ T. A. Kennedy et al., Appl. Phys. Lett. 83, 4190 (2003).

${ }^{22}$ S. Takahashi et al., Phys. Rev. Lett. 101, 047601 (2008).

${ }^{23}$ A. Gali et al., Phys. Rev. B 77, 155206 (2008).

${ }^{24}$ This form of decoherence can be found by expanding $\Omega_{n}^{(0)}$ in Eq. (7) of Ref. 11 for small angle $\theta$.

${ }^{25}$ A. Tyryshkin et al., J. Phys.: Condens. Matter 18, S783 (2006).

${ }^{26}$ W. M. Witzel and S. Das Sarma, Phys. Rev. B 74, 035322 (2006).

${ }^{27}$ V. V. Dobrovitski et al., Phys. Rev. B 77, 245212 (2008).

${ }^{28}$ A. Abragam, Principles of Nuclear Magnetism (Oxford University Press, USA, 1983).

${ }^{29}$ The magnetic field magnitude $B_{0}$ and angle $\theta$ relative to the axis of one class of NVs in the ensemble can be extracted from the ESR transition frequencies $\nu_{1}$ and $\nu_{2}$ using $B_{0}=\sqrt{\left(P-D^{2}\right) / 3}$ and $\cos ^{2} \theta=\left(Q+9 D B^{2}+2 D^{3}\right) / 27 D B^{2}$, where $D \simeq 2.87 \mathrm{GHz}$ is the zero-field splitting, $P=\nu_{1}^{2}+\nu_{2}^{2}-\nu_{1} \nu_{2}$ and $Q=\left(\nu_{1}+\nu_{2}\right)\left(2 \nu_{1}^{2}+2 \nu_{2}^{2}\right.$ $\left.-5 \nu_{1} \nu_{2}\right)$

${ }^{30}$ The angle dependence can be modeled by $\alpha=\left(c_{1}^{2}\right.$ $\left.+c_{2}^{2} \cos 2 \theta\right) \sin ^{2} \theta$, where $c_{1}$ and $c_{2}$ are parameters that depend on the hyperfine interaction between an NV center and a proximal nuclear spin. 